\title{
Pleuroparenchymal fibroelastosis associated with telomerase reverse transcriptase mutations
}

\author{
To the Editor:
}

We read with great interest the article by Newton et al. [1]. They found that mutations in the telomere maintenance machinery genes (telomerase reverse transcriptase (TERT), telomerase RNA component (TERC), regulator of telomere elongation helicase 1 and poly(A)-specific ribonuclease) led to variable interstitial lung disease (ILD) phenotypes that were universally progressive [1]. We were particularly interested by the evidence for the first time in the literature of a telomere-related gene mutation associated with pleuroparenchymal fibroelastosis (PPFE). PPFE represented eight out of the 77 (10.4\%) cases with genetic aberrations, which is intriguing compared with the rarity of disease among ILDs. Indeed, a possible familial propensity for the development of PPFE was originally suggested by our earlier report of three affected sisters [2]. According to other published studies, a family history of ILD is observed in 17-57\% of PPFE patients [3-5].

Similarly, from 2006 to 2015, 12 patients with a diagnosis of PPFE were referred to our department for further advice; of these, 10 were investigated for TERT and TERC mutations. TERT mutations were identified in five (50\%) cases (table 1). Noticeably, a patient with a history of "pulmonary fibrosis" in his sister, chronic liver disease and unexplained cardiomyopathy, was negative for TERT and TERC but was not tested for regulator of telomere elongation helicase 1 and poly(A)-specific ribonuclease. As in the article, our study included a preponderance of females (four out of five) (age 41-63 years). Three patients had a familial background of ILD, all had a low body mass index (mean $18.8 \mathrm{~kg} \cdot \mathrm{m}^{-2}$ ), and pneumothorax or pneumomediastinum occurred in four cases. The delay between ILD and PPFE diagnosis was quite long, ranging 14-45 months. High-resolution computed tomography (HRCT) was typical of PPFE in all cases, and was pathologically confirmed in four patients (three surgical lung biopsy (SLB), one lung explant). Our patients had rapidly progressive fibrosis, with a mean decline in diffusing capacity of the lung for carbon monoxide of $6.1 \%$ per year and a decline in forced vital capacity (FVC) of $3.7 \%$ per year. At the end of follow-up, two patients were transplanted without major haematological complications and one succumbed to an acute exacerbation following video-assisted thoracoscopy performed for pleurodesis and SLB. One untreated patient is doing well and the other continues to progress under pirfenidone. Regarding the non-mutated patients, one was transplanted, two had stable pulmonary function, and two died from terminal respiratory failure. There was no difference between groups in the mean yearly decline in FVC $(-3.7 \%$ in the mutated patients versus $-2.6 \%$ in the non-mutated; $\mathrm{p}=0.4$ ).

Our data are consistent with those of NEWTON et al. [1] and suggest the importance of searching telomere-related gene mutations in patients with PPFE even without a family history of ILD. These findings also raise pathogenic questions.

Interestingly, two patients presented Sjögren's syndrome. Autoimmune features have already been described in PPFE [4]. Data indicate that both telomerase activity and telomerase length are modified in various systemic immune-mediated diseases, including Sjögren's syndrome [8,9]. Accelerated telomeric erosion in immune cells resulting from inflammation could lead to premature cellular senescence and increased apoptosis, responsible for a loss of control of the immune system [8]. One may hypothesise that telomerase mutation could favour autoimmune diseases like Sjögren's syndrome.

Although pleuroparenchymal changes were typical of PPFE, with an obvious predilection for upper lobes, it is remarkable that four of our patients also demonstrated a minor interstitial fibrosis in the lower

@ERSpublications

Five cases with telomerase reverse transcriptase mutation and pleuroparenchymal fibroelastosis http://ow.ly/u33930aARaL

Cite this article as: Nunes H, Jeny F, Bouvry D, et al. Pleuroparenchymal fibroelastosis associated with telomerase reverse transcriptase mutations. Eur Respir J 2017; 49: 1602022 [https://doi.org/10.1183/ 13993003.02022-2016]. 
regions at HRCT, with a pathological pattern most reminiscent of usual interstitial pneumonia (UIP) in the lower parts at SLB in three patients. In a study of 12 PPFE patients by REDDY et al. [4], all seven cases with multiple biopsies showed a pattern of pathological involvement in the lower lobes, which was noted upon HRCT and corresponded with UIP in three cases. Similarly, in the study by ODA et al. [10], nine out of 11 patients meeting radiological criteria for the diagnosis of PPFE were histologically confirmed as

TABLE 1 Characteristics of patients with pleuroparenchymal fibroelastosis (PPFE) and reverse transcriptase (TERT) mutations

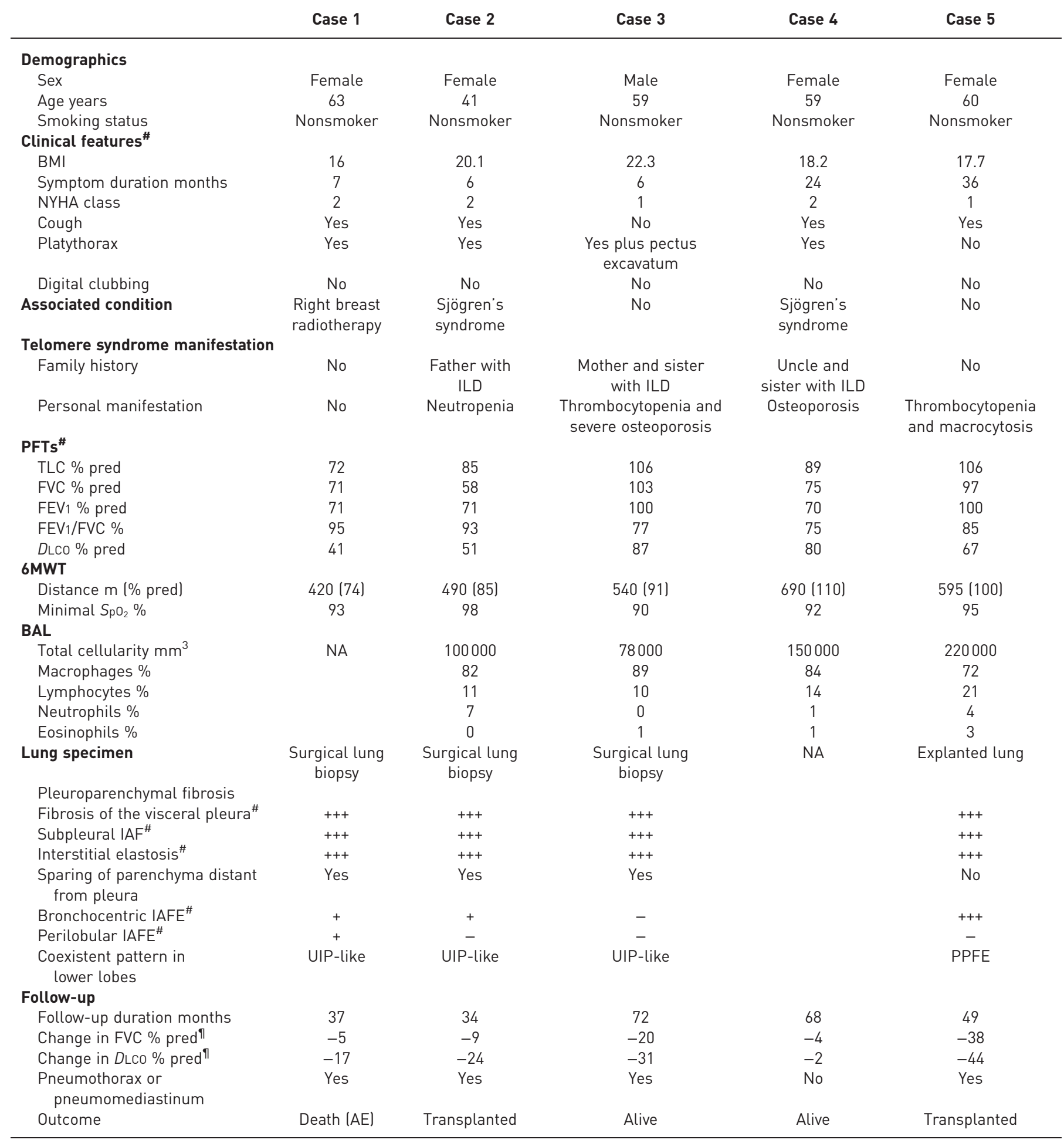


TABLE 1 Continued

Case 1

Case 2

Case 3

Case 4

Case 5

\section{Genetic findings}

Nomenclature of the TERT

variation at cDNA level and

c.3040G>C,p. c. $2159 T>C, p$.

Ala1014Pro

protein level

Nomenclature of the TERT

variation at gDNA level on

chromosome 5 (human

genome version 19)

ExAC telomerase database and other reports ${ }^{+}$

Prediction of the impact of the mutation by PolyPhen (Hum Var) $)^{\S}$

Conclusion ${ }^{f}$

Absence

Absence

0.999

0.932

VUSD

VUSD g. $1255519 C>G \quad$ g.1278883A>G

c.198_207del,p.

Ala67Profs*8

Absence

Absence

1/121392 alleles [1]

Not applicable

0.999

0.983

Path

VUSD

c. $2266 C>T, p$.

Arg756Cys

g. $1278776 \mathrm{G}>\mathrm{A}$ g.1294898_1294907del

BMI: body mass index; NYHA: New York Heart Association; ILD: interstitial lung disease; PFTs: pulmonary function tests; TLC: total lung capacity; $\%$ pred: \% predicted; FVC: forced vital capacity; FEV1: forced expiratory volume in $1 \mathrm{~s}$; DLCo: diffusing capacity of the lung for carbon monoxide; 6MWT: 6-min walk test; $\mathrm{SpO}_{2}$ : arterial oxygen saturation measured by pulse oximetry; BAL: bronchoalveolar lavage; NA: not available; IAF: intraalveolar fibrosis; IAFE: IAF and elastosis; UIP: usual interstitial pneumonia; AE: acute exacerbation; ExAC: Exome Aggregation Consortium; VUSD: variant of uncertain significance possibly disease associated; Path: pathogenic; LikePath: likely pathogenic. ${ }^{+}:$mild; $^{+++}$: severe; ${ }^{-}$: absent. \#: at presentation; ${ }^{\Re}$ : changes between last available and baseline PFTs; ${ }^{+}$: presence or absence of the TERT variation in the ExAC database (http:// exac.broadinstitute.org/l, which contains exome sequencing of about 60000 individuals, and the telomerase database (http://telomerase.asu.edu/ diseases.html\#tert), which presents the published mutations identified in telomere diseases; ${ }^{\S}$ : PolyPhen Tools available at http://genetics.bwh. harvard.edu/pph2/; ${ }^{f}$ : based on American College of Medical Genetics and Genomics recommendations [6, 7].

having PPFE with UIP, including three with a family history of ILD. However, our patients and those previously reported have shown a disease presentation clearly distinct from idiopathic pulmonary fibrosis.

It is as yet unclear whether elastosis and the more common collagen fibroproliferation are distinctive pathways of chronic scarring, and whether they may reflect discrete responses to primarily different lung damages or in differently susceptible subjects. A Brazilian group has demonstrated that elastic deposition accompanies collagen deposition in the major forms of acute and chronic idiopathic interstitial pneumonias [11]. HIROTA et al. [12] have analysed serial lung biopsies from four PPFE patients and suggested that prior interstitial inflammation or acute lung injury may be the primum movens of disease. ЕNOMOTO et al. [13] have indicated that the amount of elastic fibres is significantly reduced in the lower than in the upper lobes in PPFE as well as in UIP. Furthermore, Miele et al. [14] described a spontaneous pulmonary fibrosis in aged donkeys that shared similarities with human PPFE with a localised fibrosis in the uppermost and dorsal zones. In addition, PPFE features overlap with those of apical caps, the prevalence of which increases in the elderly and may be related to microscopic tears in tissue substructure caused by the weight of the lung itself [15].

Taken together, these data suggest that the extracellular matrix, when exposed to lung injury, responds as a whole with remodelling of all its components. Patients with telomere-related gene mutation may be predisposed to tractional injury to the peripheral lung [16]. During the extracellular matrix remodelling process, the differential degree of elastosis reaction and collagen fibroproliferation between the upper and lower lobes may be due to the fact that the lung zones are subjected to different mechanical strains, with upper development of PPFE and concomitant or subsequent development of UIP in the bases.

Hilario Nunes ${ }^{1,2}$, Florence Jeny ${ }^{1,2}$, Diane Bouvry ${ }^{2}$, Clément Picard ${ }^{3}$, Jean-François Bernaudin ${ }^{4}$, Christelle Ménard ${ }^{5,6}$, Pierre-Yves Brillet ${ }^{1,7}$, Caroline Kannengiesser ${ }^{5,6}$, Dominique Valeyre ${ }^{1,2}$ and Marianne Kambouchner ${ }^{8}$

${ }^{1}$ Université Paris 13, Sorbonne Paris Cité, EA2363 "Réponses cellulaires et fonctionnelles à l'hypoxie", Bobigny, France. ${ }^{2}$ Assistance Publique Hôpitaux de Paris, Service de Pneumologie, Hôpital Avicenne, Bobigny, France. ${ }^{3}$ Service de Pneumologie, Hôpital Foch, Suresnes, France. ${ }^{4}$ Université Pierre et Marie Curie, Paris 6, Paris, France. ${ }^{5}$ Assistance Publique Hôpitaux de Paris, Service de Génétique, Hôpital Bichat, Paris, France. ${ }^{6}$ Université Paris Diderot, Paris, France. ${ }^{7}$ Assistance Publique Hôpitaux de Paris, Service de Radiologie, Hôpital Avicenne, Bobigny, France. ${ }^{8}$ Assistance Publique Hôpitaux de Paris, Service d'Anatomie Pathologique, Hôpital Avicenne, Bobigny, France.

Correspondence: Hilario Nunes, Service de Pneumologie, Hôpital Avicenne, 125 rue de Stalingrad, 93009 Bobigny, France. E-mail: hilario.nunes@avc.aphp.fr

Received: Oct 152016 | Accepted after revision: Feb 192017

Conflict of interest: None declared. 


\section{References}

1 Newton CA, Batra K, Torrealba J, et al. Telomere-related lung fibrosis is diagnostically heterogeneous but uniformly progressive. Eur Respir J 2016; 48: 1710-1720.

2 Azoulay E, Paugam B, Heymann MF, et al. Familial extensive idiopathic bilateral pleural fibrosis. Eur Respir J 1999; 14: 971-973.

3 Shiota S, Shimizu K, Suzuki M, et al. [Seven cases of marked pulmonary fibrosis in the upper lobe]. Nihon Kokyūki Gakkai Zasshi J Jpn Respir Soc 1999; 37: 87-96.

4 Reddy TL, Tominaga M, Hansell DM, et al. Pleuroparenchymal fibroelastosis: a spectrum of histopathological and imaging phenotypes. Eur Respir J 2012; 40: 377-385.

5 Frankel SK, Cool CD, Lynch DA, et al. Idiopathic pleuroparenchymal fibroelastosis: description of a novel clinicopathologic entity. Chest 2004; 126: 2007-2013.

6 Richards S, Aziz N, Bale S, et al. Standards and guidelines for the interpretation of sequence variants: a joint consensus recommendation of the American College of Medical Genetics and Genomics and the Association for Molecular Pathology. Genet Med Off J Am Coll Med Genet 2015; 17: 405-424.

7 Borie R, Tabèze L, Thabut G, et al. Prevalence and characteristics of TERT and TERC mutations in suspected genetic pulmonary fibrosis. Eur Respir J 2016; 48: 1721-1731.

8 Georgin-Lavialle S, Aouba A, Mouthon L, et al. The telomere/telomerase system in autoimmune and systemic immune-mediated diseases. Autoimmun Rev 2010; 9: 646-651.

9 Kawashima M, Kawakita T, Maida Y, et al. Comparison of telomere length and association with progenitor cell markers in lacrimal gland between Sjögren syndrome and non-Sjögren syndrome dry eye patients. Mol Vis 2011; 17: 1397-1404.

10 Oda $\mathrm{T}$, Ogura $\mathrm{T}$, Kitamura $\mathrm{H}$, et al. Distinct characteristics of pleuroparenchymal fibroelastosis with usual interstitial pneumonia compared with idiopathic pulmonary fibrosis. Chest 2014; 146: 1248-1255.

11 Rozin GF, Gomes MM, Parra ER, et al. Collagen and elastic system in the remodelling process of major types of idiopathic interstitial pneumonias (IIP). Histopathology 2005; 46: 413-421.

12 Hirota T, Yoshida Y, Kitasato Y, et al. Histological evolution of pleuroparenchymal fibroelastosis. Histopathology 2015; 66: 545-554.

13 Enomoto N, Kusagaya H, Oyama Y, et al. Quantitative analysis of lung elastic fibers in idiopathic pleuroparenchymal fibroelastosis (IPPFE): comparison of clinical, radiological, and pathological findings with those of idiopathic pulmonary fibrosis (IPF). BMC Pulm Med 2014; 14: 91.

14 Miele A, Dhaliwal K, Du Toit N, et al. Chronic pleuropulmonary fibrosis and elastosis of aged donkeys: similarities to human pleuroparenchymal fibroelastosis. Chest 2014; 145: 1325-1332.

15 Yousem SA. Pulmonary apical cap: a distinctive but poorly recognized lesion in pulmonary surgical pathology. Am J Surg Pathol 2001; 25: 679-683.

16 Leslie KO. Idiopathic pulmonary fibrosis may be a disease of recurrent, tractional injury to the periphery of the aging lung: a unifying hypothesis regarding etiology and pathogenesis. Arch Pathol Lab Med 2012; 136: 591-600.

Copyright @ERS 2017

\section{From the authors:}

We thank H. Nunes and colleagues for their interest in our study of patients with telomere-related gene mutations associated with pleuroparenchymal fibroelastosis (PPFE) [1]. Their cohort of five patients with PPFE and rare variants in telomerase reverse transcriptase (TERT) is similar to ours with regard to its female predominance. Here we provide additional details of our eight cases to point out additional similarities and differences between these two cohorts (table 1).

A diagnosis of PPFE was made using multidisciplinary diagnosis and histopathological examination of lung tissue in seven of the eight cases in our cohort. While changes consistent with PPFE were seen in the upper lobes of computed tomography (CT) scans in four of the six cases for which high-resolution CT scans were available, the presence of lower lobe fibrosis resembling a usual interstitial pneumonia (UIP) lesion was also noted in four cases. Similar to the findings of H. Nunes and colleagues, we often found the co-existence of PPFE with UIP-like features (fibroblastic foci, honeycombing) on pathologic evaluation. The women in our cohort also had a low body mass index (mean 20.5, range 16.8-26.7). We also noted a high incidence of either spontaneous pneumothorax or pneumomediastinum (three out of eight patients), a high proportion of never-smokers (seven out of eight), and a wide age range at diagnosis (30-66 years). There was evidence of several manifestations of short telomere syndrome, including a family history of pulmonary fibrosis, macrocytosis, anaemia, thrombocytopenia, pancytopenia, myelodysplastic syndrome or transient liver function test abnormalities in six individuals. All had evidence of interstitial lung disease progression, with seven patients dying or undergoing lung transplantation.

@ERSpublications

A diagnosis of PPFE is linked to pathogenic variants in three different telomere-related genes http://ow.ly/eGOq30aPkgx

Cite this article as: Newton CA, Batra K, Torrealba J, et al. Pleuroparenchymal fibroelastosis associated with telomerase reverse transcriptase mutations. Eur Respir J 2017; 49: 1700696 [https://doi. org/10.1183/13993003.00696-2017]. 
TABLE 1 Characteristics of patients with pleuroparechymal fibroelastosis (PPFE) and pathogenic rare variants in telomerase reverse transcriptase (TERT), telomerase RNA component (TERC), and regulator of telomere elongation helicase 1 (RTEL1)

\begin{tabular}{|c|c|c|c|c|c|c|c|c|}
\hline & Case 1 & Case 2 & Case 3 & Case 4 & Case 5 & Case 6 & Case 7 & Case 8 \\
\hline Sex & Female & Male & Female & Female & Female & Female & Female & Female \\
\hline Ethnicity & Caucasian & Caucasian & Caucasian & African American & Caucasian & Caucasian & Hispanic & Caucasian \\
\hline Age at diagnosis years & 60 & 65 & 60 & 30 & 33 & 57 & 64 & 66 \\
\hline Smoking status & Never & Former & Never & Never & Never & Never & Never & Never \\
\hline BMI & 21.6 & 32 & 18.3 & 21.6 & 26.7 & 18.3 & 16.8 & 20.1 \\
\hline $\begin{array}{l}\text { Pneumothorax/ } \\
\text { pneumomediastinum }\end{array}$ & Pneumomediastinum & & & & Pneumothorax & & Pneumomediastinum & \\
\hline $\begin{array}{l}\text { Family history of } \\
\text { pulmonary fibrosis }\end{array}$ & Yes & Yes & Yes & No & Yes & Yes & Yes & Yes \\
\hline $\begin{array}{l}\text { Telomeropathy } \\
\text { manifestations }\end{array}$ & $\begin{array}{l}\text { Transient LFT } \\
\text { elevation }\end{array}$ & Macrocytosis & $\begin{array}{l}\text { Pancytopenia, } \\
\text { MDS }\end{array}$ & $\begin{array}{l}\text { Anaemia, } \\
\text { thrombocytopenia }\end{array}$ & Macrocytosis & $\begin{array}{l}\text { Anaemia, } \\
\text { macrocytosis }\end{array}$ & & Breast cancer \\
\hline Gene & RTEL 1 & RTEL 1 & TERC & TERT & TERT & TERT & TERT & TERT \\
\hline DNA change & c.2206_2208delGAC & c. $2005 \mathrm{C}>\mathrm{T}$ & r. $182 g>c$ & c. $2539 \mathrm{G}>\mathrm{A}$ & c. $416 T>G$ & c. $1892 \mathrm{G}>\mathrm{A}$ & c. $2851 C>T$ & c. $430 \mathrm{G}>\mathrm{A}$ \\
\hline Impact on protein & p.Asp736del & p.Gln669X & & p.Gly847Ser & p.Leu139Arg & pArg631Gln & p.Arg951Trp & p.Val144Met \\
\hline ExAC frequency & Absent & Absent & Absent & Absent & Absent & Absent & $8.29 \times 10^{-6}$ & Absent \\
\hline $\begin{array}{l}\text { Age-adjusted LTL } \\
\text { percentile }\end{array}$ & $<1 s t$ & & $<1$ st & $6-7$ th & $<1$ st & $<1 s t$ & & \\
\hline \multicolumn{9}{|l|}{ HRCT features } \\
\hline Upper zone & PPFE & Reticulations & & $\begin{array}{c}\text { Fibrocystic } \\
\text { changes }\end{array}$ & $\begin{array}{l}\text { PPFE with } \\
\text { reticulations }\end{array}$ & PPFE & PPFE & \\
\hline Lower zone & Reticulations & Reticulations & & $\begin{array}{l}\text { Reticulations, } \\
\text { honeycombing }\end{array}$ & PPFE & PPFE & $\begin{array}{l}\text { Reticulations, } \\
\text { honeycombing }\end{array}$ & \\
\hline Biopsy specimen & Explant & $\begin{array}{l}\text { Surgical } \\
\text { biopsy }\end{array}$ & Explant & Explant & & Explant & Surgical biopsy & Surgical biopsy \\
\hline Pathologic diagnosis & PPFE & PPFE & PPFE & PPFE & & PPFE & PPFE & PPFE \\
\hline UIP features & & $\begin{array}{l}\text { Fibroblastic } \\
\text { foci }\end{array}$ & Honeycombing & Honeycombing & & & Honeycombing & $\begin{array}{l}\text { Honeycombing, } \\
\text { fibroblastic foci }\end{array}$ \\
\hline Outcome & Transplant & Death & Transplant & Transplant & Alive & Transplant & Death & Death \\
\hline
\end{tabular}

BMI: body mass index; LFT: liver function test; MDS: myelodysplastic syndrome; ExAC: Exome Aggregation Consortium; LTL: leukocyte telomere length; HRCT: high-resolution computed tomography; UIP: usual interstitial pneumonia. 
Individuals with pathogenic variants in three different telomere-related genes (TERT, telomerase RNA component, regulator of telomere elongation helicase 1) were included in our PPFE cohort, whereas only variants in TERT were described in the cohort of $\mathrm{H}$. Nunes and colleagues. All variants were very rare with allele frequencies $<10^{-5}$. Telomere lengths were not measured by $\mathrm{H}$. Nunes and colleagues. For those individuals in our cohort in which blood leukocytes were available, the age-adjusted leukocyte telomere lengths were all $<10$ th percentile, with most $<1$ st percentile. Two of the individuals in the cohort of H. Nunes and colleagues had a diagnosis of Sjogren's syndrome. While we did not find evidence of autoimmune disease in our PPFE cohort, we did have two individuals of the 115 patients with mutations in one of four different telomere-related genes that met the diagnostic criteria for scleroderma; neither of those patients had radiographic evidence of PPFE.

We agree with $\mathrm{H}$. Nunes and colleagues that a diagnosis of PPFE should bring to mind a short telomere syndrome, especially if a positive family history of pulmonary fibrosis is obtained, given its relatively high prevalence $(10.4 \%)$ in patients with telomere-related gene mutations. Short telomere length independently predicts worse transplant-free survival in patients with sporadic idiopathic pulmonary fibrosis [2], and the presence of a telomere-related mutation is associated with poor outcome regardless of the specific diagnosis [1]. Therefore, the decline in lung function and poor survival described by $\mathrm{H}$. Nunes and colleagues may not be related to the diagnosis of PPFE, but rather due to telomere dysregulation. Further investigation is warranted to determine the aetiology of the injury and mechanisms that lead to extracellular deposition of elastin as opposed to collagen in these patients.

Chad A. Newton ${ }^{1,2}$, Kiran Batra ${ }^{3}$, Jose Torrealba ${ }^{4}$, Keith Meyer ${ }^{5}$, Ganesh Raghu ${ }^{6}$ and Christine Kim Garcia ${ }^{1,2}$ ${ }^{1}$ Eugene McDermott Centre for Human Growth and Development, University of Texas Southwestern Medical Centre, Dallas, TX, USA. ${ }^{2}$ Dept of Medicine, University of Texas Southwestern Medical Centre, Dallas, TX, USA. ${ }^{3}$ Dept of Radiology, University of Texas Southwestern Medical Centre, Dallas, TX, USA. ${ }^{4}$ Dept of Pathology, University of Texas Southwestern Medical Centre, Dallas, TX, USA. ${ }^{5}$ Dept of Medicine, University of Wisconsin, Madison, WI, USA. ${ }^{6}$ Dept of Medicine, University of Washington Seattle, Seattle, WA, USA.

Correspondence: Christine Kim Garcia, Eugene McDermott Centre for Human Growth and Development, University of Texas Southwestern Medical Centre, 5323 Harry Hines Blvd, Dallas, TX 75390-8591, USA. E-mail: christine.garcia@ utsouthwestern.edu

Received: April 032017 | Accepted: April 042017

Support statement: Funding information for this article has been deposited in the Crossref Funder Registry.

Conflict of interest: Disclosures can be found alongside this article at erj.ersjournals.com

\section{References}

1 Newton CA, Batra K, Torrealba J, et al. Telomere-related lung fibrosis is diagnostically heterogeneous but uniformly progressive. Eur Respir J 2016; 48: 1710-1720.

2 Stuart BD, Lee JS, Kozlitina J, et al. Effect of telomere length on survival in patients with idiopathic pulmonary fibrosis: an observational cohort study with independent validation. Lancet Respir Med 2014; 2: 557-565. 